\title{
Two type IV secretion systems with different functions in Burkholderia cenocepacia K56-2
}

\begin{abstract}
Correspondence
Carlos F. Gonzalez

cf-gonzalez@tamu.edu
\end{abstract}

Received 24 July 2009

Revised 2 September 2009

Accepted 3 September 2009

\author{
Ruifu Zhang, ${ }^{1} \dagger$ John J. LiPuma ${ }^{2}$ and Carlos F. Gonzalez ${ }^{1}$ \\ ${ }^{1}$ Department of Plant Pathology and Microbiology, Texas A\&M University, College Station, \\ TX 77840, USA \\ ${ }^{2}$ Department of Pediatrics and Communicable Diseases, University of Michigan Medical School, \\ Ann Arbor, MI 48109, USA
}

\begin{abstract}
Bacterial type IV secretion systems (T4SS) perform two fundamental functions related to pathogenesis: the delivery of effector molecules to eukaryotic target cells, and genetic exchange. Two T4SSs have been identified in Burkholderia cenocepacia K56-2, a representative of the ET12 lineage of the $B$. cepacia complex (Bcc). The plant tissue watersoaking (Ptw) T4SS encoded on a resident $92 \mathrm{~kb}$ plasmid is a chimera composed of VirB/D4 and F-specific subunits, and is responsible for the translocation of effector(s) that have been linked to the Ptw phenotype. The bc-VirB/D4 system located on chromosome II displays homology to the VirB/D4 T4SS of Agrobacterium tumefaciens. In contrast to the Ptw T4SS, the bc-VirB/D4 T4SS was found to be dispensable for Ptw effector(s) secretion, but was found to be involved in plasmid mobilization. The fertility inhibitor Osa did not affect the secretion of Ptw effector(s) via the Ptw system, but did disrupt the mobilization of a RSF1010 derivative plasmid.
\end{abstract}

\section{INTRODUCTION}

The Burkholderia cepacia complex (Bcc) consists of at least 17 phenotypically similar but genotypically distinct species of non-fermenting, Gram-negative bacteria that are found in a diverse set of niches (Coenye et al., 2001; Vanlaere et al., 2005). These species have a high degree of $16 \mathrm{~S}$ rDNA gene (98-100\%) and recA (94-95\%) sequence similarity, and moderate levels of DNA-DNA hybridization (30$50 \%$ ) (Coenye \& Vandamme, 2003). Members of the Bcc are opportunistic pathogens, capable of causing disease in plants, animals and particularly causing life-threatening respiratory infections in persons with cystic fibrosis (Berriatua et al., 2001; Gonzalez et al., 1997; LiPuma, 2003). Strains isolated from infected cystic fibrosis patients indicate that the distribution of species is quite disproportionate, with B. cenocepacia and Burkholderia multivorans accounting for $85-90 \%$ of infection in this patient population (LiPuma, 1998a, b).

Secretion of proteins across bacterial membranes is of fundamental importance in bacterial virulence and occurs through a variety of mechanisms, from simple onecomponent systems to complex multi-component machineries. Secretion pathways have been classified into

tPresent address: Biological and Agricultural Engineering Department, University of California, One Shields Avenue, Davis, CA 95616, USA.

Abbreviations: T4SS, type IV secretion system; Bcc, Burkholderia cepacia complex; Ptw, plant tissue watersoaking; VBG, Vogel-Bonner minimal medium amended with $1.0 \%$ glucose. seven major evolutionarily and functionally related groups, termed types I-VII (Abdallah et al., 2007; Kostakioti et al., 2005; Schell et al., 2007; Thanassi \& Hultgren, 2000). There are numerous examples of the importance of type III (Plano et al., 2001) and type IV secretion systems (T4SSs) (Christie, 2001) in the infection process for both plant and animal pathogens, with evidence that type III secretion systems are derived from flagella assembly constituents modified to function as a transport mechanism for virulence factors (Macnab, 1999). T4SSs originally evolved from bacterial conjugation systems and are functionally diverse, in terms of both the transported substrate (proteins or DNA-protein complexes) and the intended recipients, which range from micro-organisms of the same or different species to inter-kingdom transfer to fungi, plants or mammalian target cells (Cascales \& Christie, 2003).

The T4SSs have been classified into four subgroups, each of which is specialized for a specific function and contributes in a unique manner to pathogenesis: effector molecule translocation into host target cells; conjugation of chromosomal and plasmid DNA; DNA uptake and transformation; and DNA release into the extracellular milieu (Backert \& Meyer, 2006). Agrobacterium tumefaciens has three T4SSs: the virB/D4 system encoded by the Ti plasmid is the paradigm of T4SS and transfers T-DNA into plant cells, resulting in the development of crown-gall tumours (Christie et al., 2005); the trb system, required for the conjugal transfer of the Ti plasmid between cells of $A$. tumefaciens (Li et al., 1998, 1999); and the avhB system, 
which mediates the conjugal transfer of the pAtC58 cryptic plasmid (Chen et al., 2002). AvhB also promotes the conjugal transfer of the IncQ plasmid RSF1010. Helicobacter pylori possesses two T4SSs with different roles. The Cag secretory apparatus functions in the translocation of CagA into host cells, whereas the com system functions to take up DNA to facilitate genetic variation (Dhar et al., 2003; Ding et al., 2003). Legionella pneumophila has a functional T4SS designated dot/icm that is essential for pathogenesis and plasmid DNA conjugal transfer (Segal et al., 1998; Segal \& Shuman, 1998; Vogel et al., 1998). In addition, this pathogen has another T4SS distinct from dot/ $\mathrm{icm}$, designated the $l v h$ system, which is dispensable for intracellular growth in its host, only partially required for RSF1010 conjugation, and can substitute for some components of the dot/icm system in plasmid conjugation (Segal et al., 1999). Other pathogens, such as Brucella and Bartonella spp., require T4SSs to persist in the intracellular environments of their mammalian hosts (O'Callaghan et al., 1999; Schulein \& Dehio, 2002), whereas the closely related plant symbiont Sinorhizobium meliloti 1021 exploits a virB-like T4SS to transfer DNA (Jones et al., 2007).

Two T4SSs have been identified in B. cenocepacia K56-2. The plant tissue watersoaking (Ptw) T4SS, which is encoded on a resident $92 \mathrm{~kb}$ plasmid, is a chimera composed of VirB/D4 and F-specific subunits, and is responsible for the secretion of a plant cytotoxic protein(s) that causes Ptw (Engledow et al., 2004). The second T4SS, designated bc-VirB/D4, is located on chromosome II and bears homology to the VirB/D4 T4SS of A. tumefaciens, but its function is still unknown. In this report, we elucidated the functions of the two T4SSs in B. cenocepacia K56-2.

\section{METHODS}

Bacterial strains, plasmids and growth conditions. The bacterial strains and plasmids used in this study are listed in Table 1. LuriaBertani (LB) medium was used for routine maintenance of cultures. Vogel-Bonner minimal medium (Vogel \& Bonner, 1956) amended with $1.0 \%$ glucose (VBG) was used in mating experiments. $B$. cenocepacia and Escherichia coli strains were grown at $37^{\circ} \mathrm{C}$. Antibiotics were added to media at the following concentrations: $100 \mu \mathrm{g}$ trimethoprim (Tp) $\mathrm{ml}^{-1}, 20 \mu \mathrm{g}$ tetracycline (Tc) $\mathrm{ml}^{-1}, 30 \mu \mathrm{g}$ chloramphenicol $(\mathrm{Cm}) \mathrm{ml}^{-1}$, and $30 \mu \mathrm{g}$ kanamycin $(\mathrm{Km}) \mathrm{ml}^{-1}$ for $E$. coli; $200 \mu \mathrm{g} \mathrm{Tp} \mathrm{ml} l^{-1}, 200 \mu \mathrm{g} \mathrm{Tc} \mathrm{ml} l^{-1}$ and $300 \mu \mathrm{g} \mathrm{Cm} \mathrm{ml}^{-1}$ for $B$. cenocepacia K56-2. For B. multivorans TL249-Nal, VBG was amended with $0.05 \mathrm{mM}$ lysine, $500 \mu \mathrm{g}$ nalidixic acid $\mathrm{ml}^{-1}$ and $50 \mu \mathrm{g} \mathrm{Tc} \mathrm{ml} \mathrm{T}^{-1}$ for selection of plasmid transfer. The complex medium TN broth (TNB) was used for liquid cultures in plasmid-curing studies (Hansen \& Olsen, 1978). Solid medium was identical except it lacked $\mathrm{KNO}_{3}$ and was supplemented with $20 \mathrm{~g}$ agar $\mathrm{l}^{-1}$.

DNA manipulations. Genomic DNA was extracted using a DNeasy kit (Qiagen), small-scale plasmid preparation was done using the Miniprep kit (Qiagen), and gel extraction and PCR product purification were conducted using the Qiaquick gel extraction kit and Qiaquick PCR purification kit, respectively (Qiagen). All restriction endonucleases, Taq DNA polymerase and T4 DNA ligase were purchased from New England Biolabs. Oligonucleotide primers were synthesized by Operon Biotechnologies Inc. A Gene Pulser (Bio-
Rad) was used for electroporation. Southern blot analysis was conducted using the DIG High Prime DNA Labelling and Detection Starter kit, according to the manufacturer's instructions (Roche Applied Science), DNA sequences were determined in the DNA sequencing laboratory of the Institute of Plant Genomics \& Biotechnology, Texas A\&M University. Sequence analysis and alignments were conducted using Vector NTI software (Invitrogen).

Plasmid construction for allelic exchange. Since B. cenocepacia strain K56-2 is clonally related to the sequenced strain J2315 (Mahenthiralingam et al., 2000; Holden et al., 2009), PCR primers for regions flanking the bcvirD4 locus in strain K56-2 were designed based on J2315 sequences BCA0334 and BCA0336 (www.sanger. ac.uk/Projects/B_cenocepacia/). To construct plasmid pEX18Tc$\Delta$ bcvirD4:: Tp used to delete bcvirD4 from K56-2, a $1.7 \mathrm{~kb}$ fragment upstream of $b c v i r D 4$ was amplified with primers BcvirD4-up-forward (5'-CAACATCAGGGTACCGAGTT-3', KpnI site underlined) and BcvirD4-up-reverse (5'-GTACGGATCCTAGCACGAATTGCTGA-3', BamHI site underlined), and the PCR product was cloned into plasmid pEX18Tc, digested with $K p n \mathrm{I}$ and $B a m \mathrm{HI}$, to obtain plasmid pEX18Tc:: $\Delta b c v i r D 4 \mathrm{up}$. The $1.7 \mathrm{~kb}$ fragment downstream of bcvirD4 was amplified using primers BcvirD4-down-forward (5'-CATGTGCGAGCGGACTATCTGGAA-3', located next to an endogenous BamHI site) and BcvirD4-down-reverse (5'-GTTCTAGACCGACACGAATG-3', the introduced $\mathrm{XbaI}$ site is underlined). The PCR product was cloned into pEX18Tc:: $\Delta b c v i r D 4 u p$ and digested with BamHI and XbaI to obtain pEX18Tc:: $\Delta b c v i r D 4$. The Tp cassette from plasmid R388 was amplified with primers Tp-forward (5'GTGGATCCGCTTTCTGATAG-3', BamHI site underlined) and Tpreverse (5'-GAGGATCCGATTGTGAGCG-3', BamHI site underlined). The PCR product was cloned into the BamHI site of plasmid pEX18Tc:: $\Delta b c v i r D 4$, to obtain pEX18Tc- $\Delta b c v i r D 4:: T p$, which was used for allelic exchange to delete the bcvirD4 gene and introduce a selectable marker.

Genomic sequences of strain J2315 (www.sanger.ac.uk/Projects/ B_cenocepacia/) were again used to design PCR primers to amplify ptwD4 from strain K56-2. To construct plasmid pEX18Tc$\Delta p t w D 4:: \mathrm{Tp}$, used to obtain a deletion in $p t w D 4$, a $1.8 \mathrm{~kb}$ fragment upstream of $p t w D 4$ was amplified with primers PtwD4-up-forward (5'-GCGCGAATTTCGTCCAGTT- $\left.3^{\prime}\right)$ and PtwD4-up-reverse (5'ACAACTTGATCGACGGGAAG- $3^{\prime}$ ). The PCR product had EcoRI and $K p n I$ sites on the $5^{\prime}$ and $3^{\prime}$ ends, respectively, and was cloned into plasmid pEX18Tc digested with EcoRI and KpnI to obtain pEX18Tc:: $\Delta p t w D 4$-up. The $1.8 \mathrm{~kb}$ fragment downstream of $p t w D 4$ was amplified with primers PtwD4-down-forward (5'-GTCTCATATGCCGCTTCGGAA- $3^{\prime}$ ) and PtwD4-down-reverse (5'-GTCTCTAGATCAGACTTGTCAACC- $3^{\prime}$; the introduced $X b a \mathrm{I}$ site is underlined). The PCR product was first cloned into plasmid pDrive (Qiagen) to obtain plasmid pDrive:: $\Delta p t w D 4$-down, then doubledigested with $K p n \mathrm{I}$ and $X b a \mathrm{I}$. The fragment was cloned into pEX18Tc:: $\Delta p t w D 4$-up double-digested with $K p n \mathrm{I}$ and $X b a \mathrm{I}$ to obtain pEX18Tc:: $\Delta p t w D 4$. The Tp cassette was obtained by $K p n \mathrm{I}$ digestion of plasmid p34S-Tp and inserted into the KpnI site of pEX18Tc:: $\Delta p t w D 4$ to obtain pEX18Tc- $\Delta p t w D 4::$ Tp. All the constructed plasmids were confirmed by sequence analyses. All plasmid constructs were performed using E. coli DH5 $\alpha$ MCR as host. Plasmid constructs were introduced by electroporation $(25 \mu \mathrm{F}, 1.5 \mathrm{kV}, 200 \Omega)$ followed by selection on LB agar amended with appropriate antibiotic(s).

Site-directed mutagenesis. Plasmids pEX18Tc- $\Delta$ virD4::Tp and pEX18Tc- $\Delta p t w D 4:: \mathrm{Tp}$ were individually introduced into B. cenocepacia K56-2 by conjugation using triparental matings with pRK2013 as the mobilizing plasmid, and selection on VBG agar plates containing $200 \mu \mathrm{g} \mathrm{Tp} \mathrm{ml} \mathrm{m}^{-1}$ for single-crossover events. It is our experience that $s a c B$ gene selection to screen the double-crossover 
Table 1. Bacterial strains and plasmids used in this study

\begin{tabular}{|c|c|c|}
\hline Strains and plasmids & Characteristics $^{\star}$ & Reference or source \\
\hline \multicolumn{3}{|l|}{ E. coli } \\
\hline DH5 $\alpha \mathrm{MCR}$ & 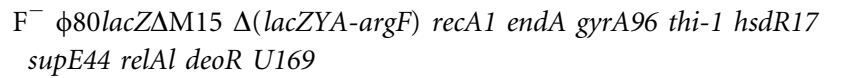 & Invitrogen \\
\hline \multicolumn{3}{|c|}{ I } \\
\hline TL249 & Soil isolate, plasmid-free, $l y s-2$ & Gaffney \& Lessie (1987) \\
\hline $\mathrm{TL} 49-\mathrm{Nal}^{\mathrm{r}}$ & Soil isolate, plasmid-free, $l y s-2, \mathrm{Nal}^{\mathrm{r}}$ & This study \\
\hline K56-2 & Cystic fibrosis respiratory isolate, $\mathrm{Ptw}^{+}$ & Sokol et al. (1999) \\
\hline RZ101 & K56-2 $\Delta$ bcvirD4:: Tp, $\mathrm{Tp}^{\mathrm{r}}$ & This study \\
\hline RZ102 & K56-2(pML122Tc) & This study \\
\hline RZ103 & RZ101(pML122Tc) & This study \\
\hline RZ104 & RZ101(pML122Tc, pBcLaR) & This study \\
\hline RZ105 & RZ101(pML122Tc, pBcLaR:: bcvirD4) & This study \\
\hline RZ106 & K56-2(pBcRLaR:: osa $)$ & This study \\
\hline RZ107 & K56-2(pML122Tc, pBcLaR) & This study \\
\hline RZ108 & K56-2(pML122Tc, pBcLaR:: osa) & This study \\
\hline \multicolumn{3}{|l|}{ Plasmids } \\
\hline pEX18Tc & Suicide vector, $s a c B, \mathrm{Tc}^{\mathrm{r}}$ & Hoang et al. (1998) \\
\hline pDrive & Cloning of PCR products, $\mathrm{Ap}^{\mathrm{r}} \mathrm{Km}^{\mathrm{r}}$ & Qiagen \\
\hline pBR325 & Source of Tc cassette, $\mathrm{Tc}^{\mathrm{r}}$ & Laboratory stock \\
\hline pRK2013 & $\mathrm{Tra}^{+} \mathrm{Mob}^{+}$ColE1 replicon, $\mathrm{Km}^{\mathrm{r}}$ & Laboratory stock \\
\hline $\mathrm{p} 34 \mathrm{~S}-\mathrm{Tp}$ & Source of $\mathrm{Tp}$ resistance cassette & Dennis \& Zylstra (1998) \\
\hline $\mathrm{pEX} 18 \mathrm{Tc}-\Delta v i r D 4:: \mathrm{Tp}$ & $\begin{array}{l}\text { pEX18Tc with virD4 upstream and downstream fragments joined by } \\
\text { Tp cassette, } \mathrm{Tc}^{\mathrm{r}} \mathrm{Tp}^{\mathrm{r}}\end{array}$ & This study \\
\hline pDrive : : bcvirD4 & bcvirD4 gene cloned into pDrive, $\mathrm{Ap}^{\mathrm{r}} \mathrm{Km}^{\mathrm{r}}$ & This study \\
\hline pBcRLaR:: bcvirD4 & bcvirD4 gene from pDrive-bcvirD4 cloned into pBcRLaR, $\mathrm{Cm}^{\mathrm{r}}$ & This study \\
\hline pDrive : : ptwD4 & ptwD4 gene cloned into pDrive, $\mathrm{Ap}^{\mathrm{r}} \mathrm{Km}^{\mathrm{r}}$ & This study \\
\hline pBcRLaR:: ptwD4 & ptwD4 gene from pDrive::ptwD4 cloned into pBcRLaR, $\mathrm{Cm}^{\mathrm{r}}$ & This study \\
\hline
\end{tabular}

${ }^{\star} \mathrm{Ap}$, ampicillin; $\mathrm{Cb}$, carbenicillin; $\mathrm{Cm}$, chloramphenicol; $\mathrm{Gm}$, gentamicin; Km, kanamycin; Nal, nalidixic acid; Tc, tetracycline; Tp, trimethoprim.

transconjugants does not function for B. cenocepacia K56-2. To screen for double-crossover events in transconjugants that had lost the vector sequence, $\mathrm{Tp}^{\mathrm{r}}$ transconjugants were picked onto both VBG agar plates containing $200 \mu \mathrm{g} \mathrm{Tp} \mathrm{ml} \mathrm{m}^{-1}$ and VBG agar plates containing $200 \mu \mathrm{g} \mathrm{Tc} \mathrm{ml}{ }^{-1}$. Transconjugants that were $\mathrm{Tp}^{\mathrm{r}} \mathrm{Tc}^{\mathrm{s}}$ were selected as target gene deletion mutants and confirmed by PCR using multiple primer set combinations, both internal and external to the target gene deletion. Deletion mutants were also confirmed by Southern blot analysis using multiple probes, including the $\mathrm{Tp}$ cassette, the deleted sequence and the amplified sequence adjacent to the target deletion.

Complementation experiments. The bcvirD4 gene was amplified using primers K56bcvirD4-forward (5'-GCTCTAGAATGAGACAGATTGC-3') and K56bcvirD4-reverse (5'-TGACTGCAGCTCA-
TTGAACACC-3'), and cloned into pDrive to obtain pDrive: :bcvirD4. The orientation of the inserted bcvirD4 gene was determined by sequencing the insert to confirm the in-frame orientation of bcvirD4. Plasmid pDrive:: bcvirD4 was then doubledigested with KpnI and HindIII, and the fragment containing bcvirD4 was gel-extracted and cloned into plasmid pBcRLaR digested with $K p n \mathrm{I}$ and HindIII to construct pBcRLaR::bcvirD4. Plasmids pBcRLaR or pBcRLaR:: bcvirD4 were individually mobilized into isolate RZ101 (bcvirD4 deletion mutant) as vector control and complementation plasmid, respectively.

ptwD4 was amplified using primers PD4-1 (5'-TGACTCAGCGAAGGAA-3') and PD4-2 (5'-ATCCGGTGGAAGCAA-3'), and the PCR product was cloned into plasmid pDrive to obtain plasmid pDrive::ptwD4. The orientation of the inserted $p t w D 4$ gene was 
determined by sequencing the insert to confirm the in-frame orientation of $p t w D 4$. Plasmid pDrive:: $p t w D 4$ was then doubledigested with BamHI and HindIII, and the fragment containing ptwD4 was gel-extracted and cloned into pBcRLaR double-digested with BamHI and HindIII to construct pBcRLaR::ptwD4. Plasmids pBcRLaR or pBcRLaR::ptwD4 were individually mobilized into isolate RZ072 (ptwD4 deletion mutant) as vector control and complementation plasmid, respectively.

Construction of pML122Tc and pBCRLaR::osa. To construct pML122Tc, the Tc cassette from pBR325 was amplified using primers TET-1 (5'-GCCATGTTTGACAGCTTATC-3' $\left.{ }^{\prime}\right)$ and TET- $2\left(5^{\prime}-\right.$ TGCGCATTCACAGTTCTCCG-3'), and cloned into pDrive. The Tc cassette was excised from pDrive by EcoRI digestion, gel-purified and cloned into the EcoRI sites of the RSF1010 derivative plasmid pML122, to obtain pML122Tc. To construct pBcRLaR:: osa, plasmid pKA165 was double-digested with BamHI and XhoI to obtain the osa fragment. The osa fragment was gel-extracted, and ligated into pBcRLaR double-digested with BamHI and XhoI to obtain pBcRLaR : : osa.

Mobilization of plasmid pML122Tc. Mobilization of plasmid pML122Tc by strain K56-2 and derivatives was determined using $B$. multivorans TL249-Nal as the recipient in biparental and triparental (using pRK2013 as helper) matings. Donor, helper and recipient strains were grown overnight at $37{ }^{\circ} \mathrm{C}$ with appropriate selection. Bacterial suspensions in LB broth were adjusted spectrophotometrically $\left(\mathrm{OD}_{600}=0.5\right)$, mixed at an equal ratio, and transferred to a positively charged sterile membrane layered on a $100 \times 15 \mathrm{~mm} \mathrm{LB}$ Petri dish containing LB agar and incubated at $37^{\circ} \mathrm{C}$. Following an $18 \mathrm{~h}$ incubation period, the cells from the matings and respective controls were washed twice in phosphate buffer $(0.125 \mathrm{M}, \mathrm{pH} 7.1)$ by centrifugation $\left(12096 \mathrm{~g}\right.$ for $10 \mathrm{~min}$ at $5{ }^{\circ} \mathrm{C}$ ). The bacterial pellets were resuspended in phosphate buffer and dilution-plated onto VBG plates amended with $0.05 \mathrm{mM}$ lysine, $500 \mu \mathrm{g}$ nalidixic acid $\mathrm{ml}^{-1}$ and $50 \mu \mathrm{g} \mathrm{Tc} \mathrm{ml}{ }^{-1}$. After $48 \mathrm{~h}$ incubation at $37^{\circ} \mathrm{C}$, all plates were evaluated. To determine the donor input (c.f.u. $\mathrm{ml}^{-1}$ ), dilutions of donor control cell suspension were plated onto VBG plates with appropriate antibiotics. Single-colony isolates of individual transconjugants were obtained by streaking on selective media. Cultures were subjected to survey lysis followed by agarose gel electrophoresis to confirm plasmid transfer.

Ptw assay. The Ptw assay was conducted as described by Engledow et al. (2004). Onion cultivar $1015 \mathrm{Y}$ was used throughout the study. Bacterial suspensions to be tested were adjusted to $\mathrm{OD}_{600}=0.5$, individual onion scales were wounded on the abaxial (inner) surface with a sterile toothpick and $10 \mu \mathrm{l}$ bacterial suspension $\left(10^{6}\right.$ c.f.u. per scale) was inoculated into the wound. Sterile double-distilled deionized water was used as negative control. Onion scales were placed on a sheet of aluminium foil that had been surface sterilized with $70 \%$ ethanol in containers containing paper towels premoistened with sterile distilled water, sealed, and incubated at $37{ }^{\circ} \mathrm{C}$. Ptw activity was assessed at $24 \mathrm{~h}$ post-inoculation by measuring the vertical and horizontal diameters of the zones.

Plasmid curing. Plasmid-curing studies were conducted essentially as described by Gonzalez et al. (1997). Briefly, cultures were grown for $18 \mathrm{~h}$ at $37^{\circ} \mathrm{C}$ in TN broth with shaking (200 r.p.m.). Bacterial cells were subcultured into prewarmed TN broth $\left(42\right.$ or $\left.44{ }^{\circ} \mathrm{C}\right)$ to a final concentration of $10^{4}$ c.f.u. $\mathrm{ml}^{-1}$ and grown with shaking (200 r.p.m.) in a water bath for $18 \mathrm{~h}$. Temperature-treated cultures were diluted and plated onto TN agar. Individual colonies were transferred onto TN agar (stock culture) and stab-inoculated onto onion scales to test for Ptw activity as described above. Isolated colonies expressing a $\mathrm{Ptw}^{-}$phenotype were purified by streaking on TN agar, and individual colonies were retested. Colonies reconfirmed as $\mathrm{Ptw}^{-}$ were subjected to plasmid survey lysis as described by Gonzalez et al. (1997).

\section{RESULTS}

\section{The bc-VirB/D4 system promotes the mobilization of plasmid pML122Tc}

We previously reported that the Ptw T4SS encoded on a resident $92 \mathrm{~kb}$ plasmid ( $\mathrm{pK} 56-2)$ is involved in the translocation of an effector(s) molecule that is responsible for the Ptw phenotype expressed by B. cenocepacia K56-2 (Engledow et al., 2004). We also identified a chromosomally encoded T4SS, which we designated the bc-VirB/D4 system (Engledow et al., 2004). Sequence analysis of this region revealed a lower $\mathrm{G}+\mathrm{C}$ content $(63 \mathrm{~mol} \%)$ compared with the $\mathrm{G}+\mathrm{C}$ content of the entire genome $(66.9 \mathrm{~mol} \%)$. Two genes, designated with the genome ORF numbers BCAM0322 and BCAM0323, upstream of the bc-VirB/D4 system showed homology to a twocomponent regulatory system (Fig. 1). A gene designated bcvirD4 showed homology to other T4SS coupling proteins, which serve as active motors necessary for substrate transfer through the T4SS (Atmakuri et al., 2003; Christie et al., 2005). To investigate the role of the chromosome-encoded bc-VirB/D4 system in B. cenocepacia K56-2, a deletion in bcvirD4 with insertion of the $\mathrm{Tp}^{\mathrm{r}}$ cassette was constructed.

Because $s a c B$ gene selection to screen for double-crossover transconjugants does not function in $B$. cenocepacia (Barrett et al., 2008; Flannagan et al., 2008), only $\mathrm{Tp}^{\mathrm{r}} \mathrm{Tc}^{\mathrm{s}}$ transconjugants were considered to be putative doublecrossover mutants. The target gene deletion was confirmed by using multiple PCR set combinations, both internal and external to the target deletion, and by Southern blot analysis using the Tp cassette and PCR products of sequence immediately downstream of bcvirD4 as probes (data not shown). The bcvirD4 deletion mutant, RZ101, caused Ptw in onion tissue-producing zones equal to that observed for K56-2 when inoculated at the same concentration (Fig. 2).

Since T4SSs play a role in recruiting and transferring plasmid DNA (Chen et al., 2002; Jones et al., 2007; Segal et al., 1998; Segal \& Shuman, 1998), we sought to determine if the bc-VirB/D4 system was involved in these functions. Plasmid pML122Tc, a derivative of the IncQ plasmid RSF1010, was introduced into K56-2 and RZ101 to obtain the strains RZ102 and RZ103, respectively. In control matings, plasmid pML122Tc was mobilized at a frequency of $1.23 \times 10^{-5}$ transconjugants per donor using donor strain RZ102, mobilizing plasmid pRK2013 and TL249-Nal as the recipient in triparental matings. In biparental matings, using only RZ102 as the donor, the mobilization of plasmid pML122Tc into the same recipient was reduced by two logs (Table 2). When the bcvirD4 deletion mutant (RZ103) was used as the donor strain, no detectable $\left(<3.57 \times 10^{-10}\right.$ transconjugants per donor $)$ 


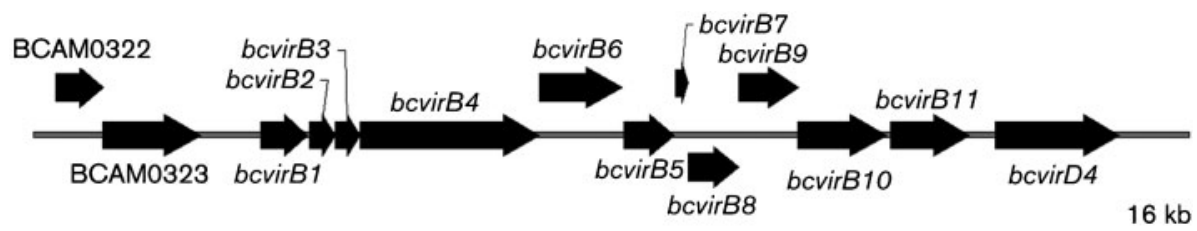

Fig. 1. Schematic representation of the bc-VirB/D4 T4SS based on the T4SS located on chromosome II of B. cenocepacia strain J2315. Designation of genes was based on homology to gene products of transfer- and translocation-related proteins. Genes are represented as solid boxes with arrowheads indicating their orientation.

transfer of pML122Tc was observed in biparental matings with TL249-Nal as the recipient. The complementation in trans of RZ103 with pBcRLaR:: bcvirD4 (RZ105) restored the ability to mobilize pML122Tc in biparental matings to approximately parental levels (Table 2). In triparental matings, pML122Tc was mobilized from strain RZ105 at parental levels (Table 2).

\section{Ptw T4SS does not mobilize plasmid pML122Tc}

We have previously reported that a functional coupling protein, PtwD4, and several other genes of the Ptw T4SS were essential to the functioning of the system involved in producing Ptw of onion tissue (Engledow et al., 2004). In the present study, we constructed a ptwD4 gene deletion mutant that circumvented potential polar effects. The deletion mutant was constructed by introducing plasmid pEX18Tc- $\Delta p t w D 4:: \mathrm{Tp}$ into K56-2 and selecting for double-crossover events. A deletion mutant, designated RZ072, was confirmed by using multiple PCR combinations both internal and external to the target deletion and by Southern blot analysis using the Tp cassette and PCR

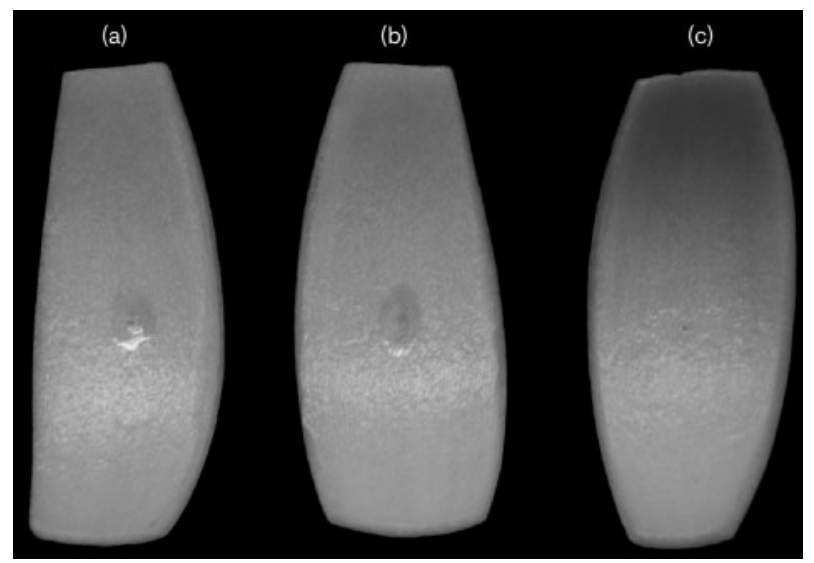

Fig. 2. Onion Ptw assay. Onion slices were wounded, inoculated with $10 \mu$ of different bacterial suspensions $\left(10^{6}\right.$ c.f.u. per scale), and incubated at $37{ }^{\circ} \mathrm{C}$ for $24 \mathrm{~h}$. (a) Strain K56-2; (b) strain $\mathrm{RZ101;} \mathrm{(c)} 10 \mu \mathrm{l}$ sterile double-distilled deionized water as negative control. products of sequence immediately downstream of the deleted ptwD4 as probes (data not shown). Strain RZ072 exhibited a $\mathrm{Ptw}^{-}$phenotype, reconfirming the role of the Ptw T4SS in the expression of the phenotype on onion tissue. Complementation of RZ072 in trans with pBcRLaR::ptwD4 (RZ074) resulted in the production of Ptw zones in plant tissue equivalent to that produced by the parental strain, K56-2 (Fig. 3).

Additionally, to determine the potential role for the Ptw T4SS in plasmid mobilization, strain RZ072 was evaluated for its ability to mobilize plasmid pML122Tc by introducing the plasmid into strain RZ072 (strain RZ075), and conducting biparental matings. The mobilization frequency of pML122Tc using donor strain RZ075 was similar to that observed for parental strain K56-2 $\left(2.42 \times 10^{-7}\right.$ vs $\left.2.44 \times 10^{-7}\right)$.

Since it would have been desirable to obtain a plasmid-free derivative of strain K56-2 to confirm the results observed with the ptwD4 deletion mutant, experiments to cure plasmid pK56-2 were conducted. Over 5000 colonies from heat-treated cultures were stab-inoculated onto onion scales to evaluate their ability to cause Ptw. Growth of K56-2 at temperatures that were successful in curing a $200 \mathrm{~kb}$ PehA-encoding plasmid harboured by B. cepacia strain ATCC 25416 (Gonzalez et al., 1997) did not result in loss of pK56-2 from strain K56-2 (data not shown).

Table 2. Mobilization of plasmid pML122Tc by K56-2 and derivatives

B. multivorans TL249-Nal was used as recipient in all assays. The results are the means of three independent experiments.

\begin{tabular}{|ccc|}
\hline $\begin{array}{c}\text { Donor } \\
\text { strains }\end{array}$ & $\begin{array}{c}\text { Transfer frequency for } \\
\text { triparental mating per } \\
\text { transconjugants donor }\end{array}$ & $\begin{array}{c}\text { Transfer frequency for } \\
\text { biparental mating per } \\
\text { transconjugant donor }\end{array}$ \\
\hline RZ102 & $1.23 \times 10^{-5}$ & $2.44 \times 10^{-7}$ \\
RZ103 & $0.76 \times 10^{-5}$ & $<3.57 \times 10^{-10}$ \\
RZ104 & $0.60 \times 10^{-5}$ & $<4.17 \times 10^{-10}$ \\
RZ105 & $1.01 \times 10^{-5}$ & $1.95 \times 10^{-7}$ \\
\hline
\end{tabular}

${ }^{\star}$ Helper plasmid pRK2013 was used in triparental matings. 


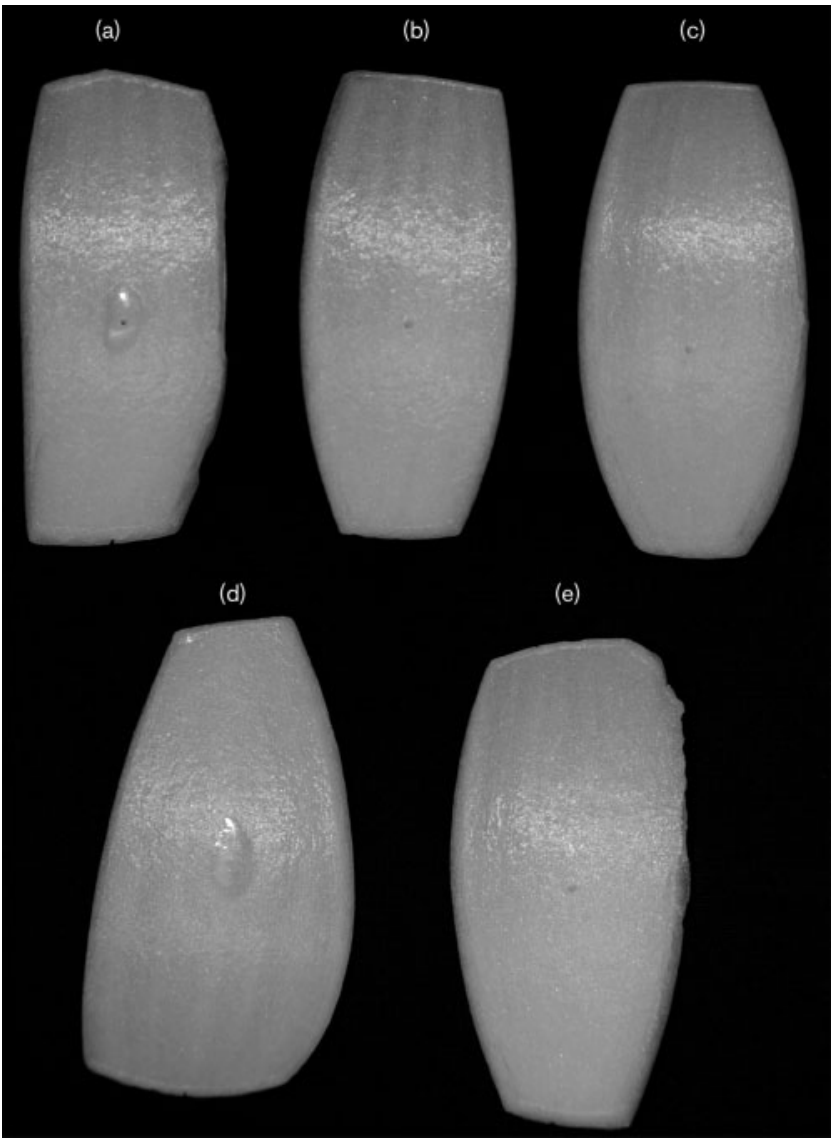

Fig. 3. Onion Ptw assay. Onion slices were wounded, inoculated with $10 \mu$ of different bacterial suspensions $\left(10^{6}\right.$ c.f.u. per scale), and incubated at $37{ }^{\circ} \mathrm{C}$ for $24 \mathrm{~h}$. (a) Strain K56-2; (b) strain RZ072; (c) strain RZ073; (d) strain RZ074; (e) $10 \mu \mathrm{l}$ sterile double-distilled deionized water as negative control.

\section{Effect of pML122Tc and Osa on effector molecule translocation via the Ptw T4SS system}

Plasmid RSF1010 has been show to interrupt T-DNA translocation via the VirB/D4 secretion channel and in the L. pneumophila icm/dot system (Cascales et al., 2005; Segal \& Shuman, 1998). To determine if the RSF1010 derivative plasmid pML122Tc could interfere with the secretion of effector protein(s) translocated via the Ptw system and attenuate the virulence of $\mathrm{K} 56-2$ in onion tissue, we compared the amount of Ptw of strain RZ102 (K56-2 harbouring pML122Tc) and wild-type K56-2. Both strains produced Ptw zones of similar diameter when inoculated on onion tissue at the same rate (data not shown).

Osa, encoded on plasmid pSa (IncW) and related in sequence to the FiwA fertility inhibition factor of plasmid $\mathrm{RP} 1(\mathrm{IncP} \alpha)$, is sufficient for suppressing oncogenicty of $A$. tumefaciens. Osa competes with VirB/D4 system substrates, such as T-DNA and VirE2, to bind to the coupling protein (Cascales et al., 2005; Lee et al., 1999; Lee \& Gelvin, 2004).
To determine the effect of Osa on the translocation of Ptw protein(s) by the Ptw system, we cloned osa into pBcRLaR, and introduced pBcRLaR : : osa into K56-2 to obtain strain RZ106. Strain RZ106 produced Ptw zones on onion tissue similar to that observed for the parental strain K56-2 control (data not shown).

\section{Effect of Osa on bc-VirB/D4 system-dependent mobilization of pML122Tc}

Osa interferes with transfer of IncQ and IncW plasmids when co-resident (Chen \& Kado, 1994; Fong \& Stanisich, 1989). More recently, it has been determined that the RSF1010 transfer intermediate and Osa suppress $A$. tumefaciens oncogenesis specifically by interfering with $\mathrm{T}$ DNA and VirE2 substrate binding to the VirD4 receptor (Cascales et al., 2005). The similarity between the $A$. tumefaciens VirB/D4 system and the chromosomally encoded bc-VirB/D4 system prompted us to determine if Osa could interfere with the mobilization of pML122Tc in B. cenocepacia. Co-residency of pBcRLaR::osa and pML122Tc in strain K56-2 (donor strain RZ108) resulted in no detectable mobilization $\left(<2.34 \times 10^{-10}\right.$ transconjugants per donor) of pML122Tc in biparental matings using TL249-Nal as the recipient, whereas mobilization of pML122Tc was observed at slightly lower levels $\left(0.73 \times 10^{-7}\right.$ transconjugants per donor $)$ for donor strain RZ107 harbouring the vector control pBcRLaR compared to that observed for K56-2 harbouring pML122Tc in biparental matings $\left(2.44 \times 10^{-7}\right.$ transconjugants per donor).

\section{DISCUSSION}

Previously, we investigated the $B$. cenocepacia plasmidencoded Ptw T4SS (Engledow et al., 2004). In that study, we determined that the Ptw T4SS, a chimera composed of VirB/D4 and F-specific subunits, is responsible for the production of the plant-disease-associated Ptw phenotype and the translocation of Ptw effector protein(s). The observations that the Ptw T4SS is involved in the translocation of a protein(s), does not contain the components necessary to support conjugation, and contains no oriT homologue indicate that functionally it is a member of the effector-translocator subfamily of T4SSs.

In that same study we identified a second gene cluster on chromosome II that showed homology to the VirB/D4 T4SS of A. tumefaciens with respect to arrangement and gene product similarity (Fig. 1) (Engledow et al., 2004). The presence of a second T4SS in B. cenocepacia is not unprecedented, since other species, such as $H$. pylori, A. tumefaciens and Bartonella henselae, have been found to harbour multiple secretion systems (Chen et al., 2002; Dhar et al., 2003; Li et al., 1998, 1999; Schröder \& Lanka, 2005). Our results indicate that the two T4SSs present in $B$. cenocepacia K56-2 do not appear to have redundant functions. Analyses of the available annotated sequenced 
genomes of B. cenocepacia AU1054 and HI2424, Burkholderia vietnamiensis $\mathrm{G} 4$ and Burkholderia xenovorans LB 400 did not reveal the presence of a Ptw-like T4SS in these strains. However, each of these strains contained a VirB/D4-like T4SS (accession numbers CP000379, CP000459, CP000616 and CP000272, respectively).

T4SSs are used by bacteria to translocate DNA and protein macromolecules to a diverse range of bacterial and eukaryotic cells (Christie et al., 2005). T4SSs also mediate horizontal gene transfer, and contribute to genome plasticity and the evolution of pathogens through dissemination of antibiotic resistance and virulence genes (Juhas et al., 2007). The A. tumefaciens VirB/D4 T4SS that transfers oncogenic T-DNA to various eukaryotic cells, as a derivative of plasmid conjugation systems, can also transfer certain mobilizable plasmids and bacterial proteins like VirE2 and VirF (Christie et al., 2005; Guo et al., 2007). In A. tumefaciens, the RSF1010 transfer intermediate and the Osa protein of plasmid pSa (IncW) render A. tumefaciens host cells nearly avirulent by interfering specifically with VirD4 receptor function by inhibiting docking of DNA and protein substrates to the translocation apparatus (Cascales et al., 2005). Our results indicate that neither the RSF1010 derivative plasmid pML122Tc nor the Osa protein affects effector protein translocation via the Ptw T4SS. The VirD4 homologue PtwD4 is the coupling protein in the Ptw T4SS, and insertions (Engledow et al., 2004) or deletions in ptwD4 (present study) disrupt translocation of the Ptw effectors. Since pML122 transfer intermediates and the Osa protein specifically interfere with VirD4 receptor function in the $A$. tumefaciens VirB/D4 T4SS, they are unlikely to be substrates for the PtwD4 coupling protein. This is most probably due to specificity of the coupling protein for the Ptw effector(s) as the coupling protein functions as a gate to mating-pair formation channels (Schröder \& Lanka, 2005). Several studies indicate that the T4SS coupling protein (i.e. VirD4 and TraG-like proteins) is the primary factor that determines the specificity of the secretion system for a particular substrate (Cabezon et al., 1994; Hamilton et al., 2000; Llosa et al., 2003). A C-terminal signal mediating the translocation of T4SS protein substrates has been identified in the VirB/ VirD4 systems of A. tumefaciens and B. henselae, and in the Dot/Icm system of L. pneumophila (Atmakuri et al., 2003; Nagai et al., 2005; Schulein et al., 2005; Simone et al., 2001; Vergunst et al., 2005). It has been proposed that these secretion systems evolved from conjugation systems and that the C-terminal signal of their secreted protein substrates originates from a relaxase ancestor (Schulein et al., 2005). It is highly likely that co-evolution of the PtwD4 coupling protein and the T4SS signal has occurred and that a specific signal mediates protein-protein interaction to initiate transfer of the Ptw effector(s) by the Ptw T4SS.

While a deletion in ptwD4 resulted in the loss of the Ptw phenotype in $\mathrm{K} 56-2$, it did not disrupt the ability to mobilize plasmid pML122Tc, since mobilization frequencies of the plasmid using donor strain RZ075 were similar to that observed for parental strain K56-2 $\left(2.42 \times 10^{-7}\right.$ vs
$2.44 \times 10^{-7}$ ) in biparental matings. The bc-VirB/D4 T4SS in B. cenocepacia K56-2 exhibited the ability to promote the transfer of the IncQ plasmid RSF1010 derivative pML122Tc (Table 2). Osa inhibits conjugative transfer of pML122 to agrobacterial recipients by interfering with pML122 binding to the VirD4 receptor without further negative effects on substrate binding to the VirB subunits (Cascales et al., 2005; Lee \& Gelvin, 2004). The observed inhibitory effect of Osa with agrobacterial recipients was approximately one log reduction in conjugative transfer frequency and $65 \%$ reduction in pML122 binding to the VirD4 receptor (Cascales et al., 2005). In the Burkholderia system, the presence of Osa reduced the transfer of pML122Tc below a detectable level $\left(<2.34 \times 10^{-10}\right.$ transconjugants per donor) when supplied in trans by plasmid pBcRLaR::osa, therefore reducing the mobilization frequency by over two logs. We observed that the mobilization frequency of pML122Tc was reduced slightly when donor strain RZ107 harbouring the vector control pBcRLaR was used in biparental matings. No detectable mobilization of pBcRLaR was observed in biparental matings.

We have reported previously that the plasmid-encoded Ptw T4SS plays a role in the intracellular survival of $B$. cenocepacia in both professional and non-professional phagocytes (Sajjan et al., 2008). A functional plasmidencoded T4SS contributes to the ability of B. cenocepacia to evade endocytic degradation, and to survive and replicate in both airway epithelial cells and monocyte-derived macrophages. In B. cenocepacia $\mathrm{K} 56-2$, it is unknown if gene products from the Ptw system substitute for proteins in the bc-VirB/D4 system, as has been observed in $L$. pneumophila where components of the dot/icm and lvh T4SSs are able to interact with one another, with lvh gene products being able to substitute for some components of the dot/icm system for RSF1010 conjugation (Segal et al., 1999). We do, however, know that the ptwD4 deletion was not complemented by bcvirD4 and vice versa, and that the Ptw system is not involved in mobilization of pML122-Tc. Although both coupling proteins contain P-loop and Walker B sites, which are characteristic of T4 secretion ATP-binding proteins (Walker et al., 1982), they show low protein sequence similarity. Further studies will identify the structural gene(s) for the Ptw system watersoaking effector protein(s) and the substrate for the bcVirB/D4 involved in DNA mobilization.

\section{ACKNOWLEDGEMENTS}

We are grateful to Peter J. Christie for providing plasmids pKA165 and pML122. This work was supported by grant GONZAL06G0 from the Cystic Fibrosis Foundation.

\section{REFERENCES}

Abdallah, A. M., Gey van Pittius, N. C., Champion, P. A., Cox, J., Luirink, J., Vandenbroucke-Grauls, C. M., Appelmelk, B. J. \& Bitter, 
W. (2007). Type VII secretion - mycobacteria show the way. Nat Rev Microbiol 5, 883-891.

Atmakuri, K., Ding, Z. \& Christie, P. J. (2003). VirE2, a type IV secretion substrate, interacts with the VirD4 transfer protein at cell poles of Agrobacterium tumefaciens. Mol Microbiol 49, 1699-1713.

Backert, S. \& Meyer, T. F. (2006). Type IV secretion systems and their effectors in bacterial pathogenesis. Curr Opin Microbiol 9, 207-217.

Barrett, A. R., Kang, Y., Inamasu, K. S., Son, M. S., Vukovich, J. M. \& Hoang, T. T. (2008). Genetic tools for allelic replacement in Burkholderia species. Appl Environ Microbiol 74, 4498-4508.

Berriatua, E., Ziluaga, I., Miguel-Virto, C., Uribarren, P., Juste, R., Laevens, S., Vandamme, P. \& Govan, J. R. (2001). Outbreak of subclinical mastitis in a flock of dairy sheep associated with Burkholderia cepacia complex infection. J Clin Microbiol 39, 990-994.

Cabezon, E., Lanka, E. \& de la Cruz, F. (1994). Requirements for mobilization of plasmids RSF1010 and ColE1 by the IncW plasmid R388: trwB and RP4 traG are interchangeable. J Bacteriol 176, 44554458.

Cascales, E. \& Christie, P. J. (2003). The versatile bacterial type IV secretion systems. Nat Rev Microbiol 1, 137-149.

Cascales, E., Atmakuri, K., Liu, Z., Binns, A. N. \& Christie, P. J. (2005). Agrobacterium tumefaciens oncogenic suppressors inhibit T-DNA and VirE2 protein substrate binding to the VirD4 coupling protein. Mol Microbiol 58, 565-579.

Chen, C. Y. \& Kado, C. I. (1994). Inhibition of Agrobacterium tumefaciens oncogenicity by the osa gene of pSa. J Bacteriol 176, 56975703.

Chen, L., Chen, Y., Wood, D. W. \& Nester, E. W. (2002). A new type IV secretion system promotes conjugal transfer in Agrobacterium tumefaciens. J Bacteriol 184, 4838-4845.

Christie, P. J. (2001). Type IV secretion: intercellular transfer of macromolecules by systems ancestrally related to conjugation machines. Mol Microbiol 40, 294-305.

Christie, P. J., Atmakuri, K., Krishnamoorthy, V., Jakubowski, S. \& Cascales, E. (2005). Biogenesis, architecture, and function of bacterial type IV secretion systems. Annu Rev Microbiol 59, 451-485.

Coenye, T. \& Vandamme, P. (2003). Diversity and significance of Burkholderia species occupying diverse ecological niches. Environ Microbiol 5, 719-729.

Coenye, T., Vandamme, P., Govan, J. R. \& LiPuma, J. J. (2001). Taxonomy and identification of the Burkholderia cepacia complex. J Clin Microbiol 39, 3427-3436.

Dennis, J. J. \& Zylstra, G. J. (1998). Plasposons: modular self-cloning minitransposon derivatives for rapid genetic analysis of Gramnegative bacterial genomes. Appl Environ Microbiol 64, 2710-2715.

Dhar, S. K., Soni, R. K., Das, B. K. \& Mukhopadhyay, G. (2003). Molecular mechanism of action of major Helicobacter pylori virulence factors. Mol Cell Biochem 253, 207-215.

Ding, Z., Atmakuri, K. \& Christie, P. J. (2003). The outs and ins of bacterial type IV secretion substrates. Trends Microbiol 11, 527-535.

Engledow, A. S., Medrano, E. G., Mahenthiralingam, E., LiPuma, J. J. \& Gonzalez, C. F. (2004). Involvement of a plasmid-encoded type IV secretion system in the plant tissue watersoaking phenotype of Burkholderia cenocepacia. J Bacteriol 186, 6015-6024.

Flannagan, R. S., Linn, T. \& Valvano, M. A. (2008). A system for the construction of targeted unmarked gene deletions in the genus Burkholderia. Environ Microbiol 10, 1652-1660.

Fong, S. T. \& Stanisich, V. A. (1989). Location and characterization of two functions on RP1 that inhibit the fertility of the IncW plasmid R388. J Gen Microbiol 135, 499-502.
Gaffney, T. D. \& Lessie, T. G. (1987). Insertion-sequence-dependent rearrangements of Pseudomonas cepacia plasmid pTGL1. J Bacteriol 169, 224-230.

Gonzalez, C. F., Pettit, E. A., Valadez, V. A. \& Provin, E. M. (1997). Mobilization, cloning, and sequence determination of a plasmidencoded polygalacturonase from a phytopathogenic Burkholderia (Pseudomonas) cepacia. Mol Plant Microbe Interact 10, 840-851.

Guo, M., Jin, S., Sun, D., Hew, C. L. \& Pan, S. Q. (2007). Recruitment of conjugative DNA transfer substrate to Agrobacterium type IV secretion apparatus. Proc Natl Acad Sci U S A 104, 20019-20024.

Hamilton, C. M., Lee, H., Li, P. L., Cook, D. M., Piper, K. R., von Bodman, S. B., Lanka, E., Ream, W. \& Farrand, S. K. (2000). TraG from RP4 and TraG and VirD4 from Ti plasmids confer relaxosome specificity to the conjugal transfer system of pTiC58. J Bacteriol 182, 1541-1548.

Hansen, J. B. \& Olsen, R. H. (1978). Isolation of large bacterial plasmids and characterization of the P2 incompatibility group plasmids pMG1 and pMG5. J Bacteriol 135, 227-238.

Hoang, T. T., Karkhoff-Schweizer, R. R., Kutchma, A. J. \& Schweizer, H. P. (1998). A broad-host-range Flp-FRT recombination system for site-specific excision of chromosomally-located DNA sequences: application for isolation of unmarked Pseudomonas aeruginosa mutants. Gene 212, 77-86.

Holden, M. T., Seth-Smith, H. M., Crossman, L. C., Sebaihia, M., Bentley, S. D., Cerdeño-Tárraga, A. M., Thomson, N. R., Bason, N., Quail, M. A. \& other authors (2009). The genome of Burkholderia cenocepacia J2315, an epidemic pathogen of cystic fibrosis patients. J Bacteriol 191, 261-277.

Jones, K. M., Lloret, J., Daniele, J. R. \& Walker, G. C. (2007). The type IV secretion system of Sinorhizobium meliloti strain 1021 is required for conjugation but not for intracellular symbiosis. J Bacteriol 189, 2133-2138.

Juhas, M., Crook, D. W., Dimopoulou, I. D., Lunter, G., Harding, R. M., Ferguson, D. J. P. \& Hood, D. W. (2007). Novel type IV secretion system involved in propagation of genomic islands. J Bacteriol 189, 761-771.

Kostakioti, M., Newman, C. L., Thanassi, D. G. \& Stathopoulos, C. (2005). Mechanisms of protein export across the bacterial outer membrane. J Bacteriol 187, 4306-4314.

Labes, M., Puhler, A. \& Simon, R. (1990). A new family of RSF1010derived expression and lac-fusion broad-host-range vectors for Gramnegative bacteria. Gene 89, 37-46.

Lee, L. Y. \& Gelvin, S. B. (2004). Osa protein constitutes a strong oncogenic suppression system that can block vir-dependent transfer of IncQ plasmids between Agrobacterium cells and the establishment of IncQ plasmids in plant cells. J Bacteriol 186, 7254-7261.

Lee, L. Y., Gelvin, S. B. \& Kado, C. I. (1999). pSa causes oncogenic suppression of Agrobacterium by inhibiting VirE2 protein export. J Bacteriol 181, 186-196.

Li, P. L., Everhart, D. M. \& Farrand, S. K. (1998). Genetic and sequence analysis of the pTiC58 trb locus, encoding a mating-pair formation system related to members of the type IV secretion family. J Bacteriol 180, 6164-6172.

Li, P. L., Hwang, I., Miyagi, H., True, H. \& Farrand, S. K. (1999). Essential components of the Ti plasmid trb system, a type IV macromolecular transporter. J Bacteriol 181, 5033-5041.

LiPuma, J. J. (1998a). Burkholderia cepacia epidemiology and pathogenesis: implications for infection control. Curr Opin Pulm Med 4, 337-341.

LiPuma, J. J. (1998b). Burkholderia cepacia. Management issues and new insights. Clin Chest Med 19, 473-486. 
LiPuma, J. J. (2003). Burkholderia cepacia complex as human pathogens. J Nematol 35, 212-217.

Llosa, M., Zunzunegui, S. \& de la Cruz, F. (2003). Conjugative coupling proteins interact with cognate and heterologous VirB10-like proteins while exhibiting specificity for cognate relaxosomes. Proc Natl Acad Sci U S A 100, 10465-10470.

Macnab, R. M. (1999). The bacterial flagellum: reversible rotary propellor and type III export apparatus. J Bacteriol 181, 7149-7153.

Mahenthiralingam, E., Coenye, T., Chung, J. W., Speert, D. P., Govan, J. R., Taylor, P. \& Vandamme, P. (2000). Diagnostically and experimentally useful panel of strains from the Burkholderia cepacia complex. J Clin Microbiol 38, 910-913.

Nagai, H., Cambronne, E. D., Kagan, J. C., Amor, J. C., Kahn, R. A. \& Roy, C. R. (2005). A C-terminal translocation signal required for Dot/ Icm-dependent delivery of the Legionella RalF protein to host cells. Proc Natl Acad Sci U S A 102, 826-831.

O'Callaghan, D., Cazevieille, C., Allardet-Servent, A., Boschiroli, M. L., Bourg, G., Foulongne, V., Frutos, P., Kulakov, Y. \& Ramuz, M. (1999). A homologue of the Agrobacterium tumefaciens VirB and Bordetella pertussis Ptl type IV secretion systems is essential for intracellular survival of Brucella suis. Mol Microbiol 33, 1210-1220.

Plano, G. V., Day, J. B. \& Ferracci, F. (2001). Type III export: new uses for an old pathway. Mol Microbiol 40, 284-293.

Sajjan, S. U., Carmody, L. A., Gonzalez, C. F. \& LiPuma, J. J. (2008). A type IV secretion system contributes to intracellular survival and replication of Burkholderia cenocepacia. Infect Immun 76, 5447-5455.

Schell, M. A., Ulrich, R. L., Ribot, W. J., Brueggemann, E. E., Hines, H. B., Chen, D., Lipscomb, L., Kim, H. S., Mrázek, J. \& other authors (2007). Type VI secretion is a major virulence determinant in Burkholderia mallei. Mol Microbiol 64, 1466-1485.

Schröder, G. \& Lanka, E. (2005). The mating pair formation system of conjugative plasmids - a versatile secretion machinery for transfer of proteins and DNA. Plasmid 54, 1-25.

Schulein, R. \& Dehio, C. (2002). The VirB/VirD4 type IV secretion system of Bartonella is essential for establishing intraerythrocytic infection. Mol Microbiol 46, 1053-1067.

Schulein, R., Guye, P., Rhomberg, T. A., Schmid, M. C., Schroder, G., Vergunst, A. C., Carena, I. \& Dehio, C. (2005). A bipartite signal mediates the transfer of type IV secretion substrates of Bartonella henselae into human cells. Proc Natl Acad Sci U S A 102, 856-861.

Segal, G. \& Shuman, H. A. (1998). Intracellular multiplication and human macrophage killing by Legionella pneumophila are inhibited by conjugal components of IncQ plasmid RSF1010. Mol Microbiol 30, 197-208.

Segal, G., Purcell, M. \& Shuman, H. A. (1998). Host cell killing and bacterial conjugation require overlapping sets of genes within a $22-\mathrm{kb}$ region of the Legionella pneumophila genome. Proc Natl Acad Sci U S A 95, 1669-1674.

Segal, G., Russo, J. J. \& Shuman, H. A. (1999). Relationships between a new type IV secretion system and the $i \mathrm{~cm} /$ dot virulence system of Legionella pneumophila. Mol Microbiol 34, 799-809.

Simone, M., McCullen, C. A., Stahl, L. E. \& Binns, A. N. (2001). The carboxy-terminus of VirE2 from Agrobacterium tumefaciens is required for its transport to host cells by the virB-encoded type IV transport system. Mol Microbiol 41, 1283-1293.

Sokol, P. A., Darling, P., Woods, D. E., Mahenthiralingam, E. \& Kooi, C. (1999). Role of ornibactin biosynthesis in the virulence of Burkholderia cepacia: characterization of $p v d A$, the gene encoding L-ornithine N(5)-oxygenase. Infect Immun 67, 4443-4455.

Thanassi, D. G. \& Hultgren, S. J. (2000). Multiple pathways allow protein secretion across the bacterial outer membrane. Curr Opin Cell Biol 12, 420-430.

Vanlaere, E., Coenye, T., Samyn, E., Van den Plas, C., Govan, J., De Baets, F., De Boeck, K., Knoop, C. \& Vandamme, P. (2005). A novel strategy for the isolation and identification of environmental Burkholderia cepacia complex bacteria. FEMS Microbiol Lett 249, 303-307.

Vergunst, A. C., van Lier, M. C., den Dulk-Ras, A., Stuve, T. A., Ouwehand, A. \& Hooykaas, P. J. (2005). Positive charge is an important feature of the C-terminal transport signal of the VirB/D4translocated proteins of Agrobacterium. Proc Natl Acad Sci U S A 102, 832-837.

Vogel, H. J. \& Bonner, D. M. (1956). Acetylornithinase of Escherichia coli: partial purification and some properties. J Biol Chem 218, 97106.

Vogel, J. P., Andrews, H. L., Wong, S. K. \& Isberg, R. R. (1998). Conjugative transfer by the virulence system of Legionella pneumophila. Science 279, 873-876.

Walker, J. E., Saraste, M., Runswick, M. J. \& Gay, N. J. (1982). Distantly related sequences in the alpha- and beta-subunits of ATP synthase, myosin, kinases and other ATP-requiring enzymes and a common nucleotide binding fold. EMBO J 1, 945-951.

Edited by: L. S. Frost 